EPJ Web of Conferences 33, 02003 (2012)

DOI: $10.1051 /$ epjconf/20123302003

(C) Owned by the authors, published by EDP Sciences, 2012

\title{
Luminescent Solar Concentrators - a low cost photovoltaics alternative
}

\author{
W.G.J.H.M. van Sark ${ }^{\mathrm{a}}$ \\ Copernicus Institute, Utrecht University, Budapestlaan 6, 3584 CD Utrecht, the Netherlands
}

\begin{abstract}
Luminescent solar concentrators (LSCs) are being developed as a potentially low cost-per-Wp photovoltaic device, suited for applications especially in the built environment. LSCs generally consist of transparent polymer sheets doped with luminescent species, either organic dye molecules or semiconductor nanocrystals. Direct and diffuse incident sunlight is absorbed by the luminescent species and emitted at redshifted wavelengths with high quantum efficiency. Optimum design ensures that a large fraction of emitted light is trapped in the sheet, which travels to the edges where it can be collected by one or more mono- or bifacial solar cells, with minimum losses due to absorption in the sheet and re-absorption by the luminescent species. Today's record efficieny is $7 \%$, however, $10-15 \%$ is within reach. Optimized luminescent solar concentrators potentially offer lower cost per unit of power compared to conventional solar cells. Moreover, LSCs have an increased conversion efficiency for overcast and cloudy sky conditions, having a large fraction of diffuse irradiation, which is blueshifted compared to clear sky conditions. As diffuse irradiation conditions are omnipresent throughout mid- and northern-European countries, annual performance of LSCs is expected to be better in terms of $\mathrm{kWh} / \mathrm{Wp}$ compared to conventional PV.
\end{abstract}

\section{Introduction}

The key driver in global solar photovoltaic energy technology today still is lowering the cost per unit of power generated, in which fundamental research toward reaching high conversion efficiencies is going hand in hand with research in lowering production cost. Enormous progress has been made in the past decades, leading to cumulative installed capacity of $67.4 \mathrm{GW}$ worldwide at the end of 2011 [1] and module cost between 0.8-2 Euro/Wp, depending on technology. This already has brought electricity cost close to the price of $\sim 0.2$ Euro/kWh consumers pay today for some European countries, but is still far off from bulk electricity generation cost of $\sim 0.04$ Euro/kWh for conventional, fossil-fuel based power plants.

One of the major limitations to the conversion efficiency of solar cells is the limited use of the solar spectrum, leading to maximum theoretical efficiency of $\sim 30 \%$ for single junction crystalline silicon solar cells. Conversion of the incident solar spectrum to monochromatic light would greatly increase the conversion efficiency. Down shifting was suggested in the late 1970s to be used in so-

\footnotetext{
a e-mail : w.g.j.h.m.vansark@uu.nl
} 
called luminescent solar concentrators (LSC) $[3,4]$. To these LSCs one or more solar cell(s) were attached. LSCs consist of a highly transparent plastic, in which luminescent species, usually organic dye molecules, are dispersed, see Fig. 1. These dyes absorb incident light and emit it at a red-shifted wavelength, with high quantum efficiency. Internal reflection ensures collection of part of the
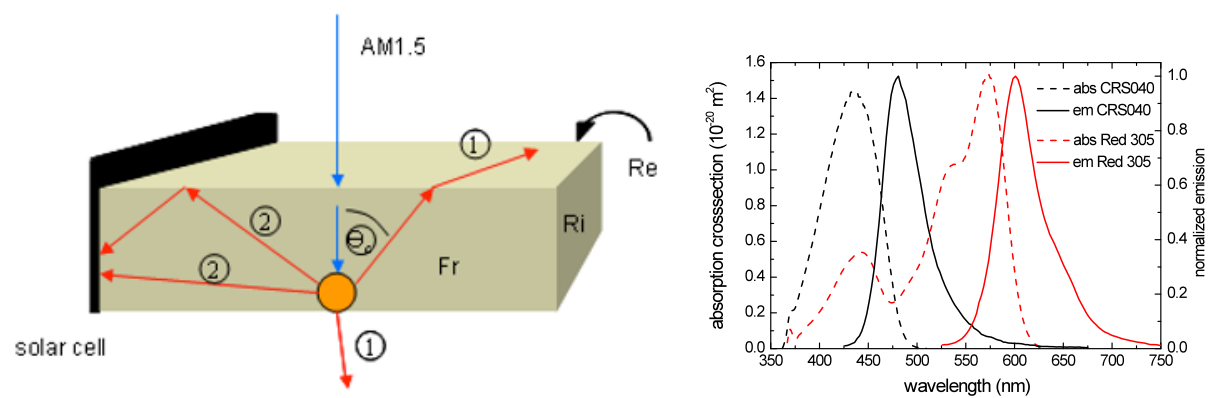

Fig. 1. (left) Schematic 3D view of a luminescent concentrator. AM1.5 light is incident on the top. The light is absorbed by a luminescent particle, and its luminescence is randomly emitted. Part of the emission falls within the escape cone and is lost from the luminescent concentrator at the surfaces (1).

The other part (2) is guided to the solar cell by total internal reflection [2]. (right) Absorption crosssection and normalized emission spectra of two fluorescent dyes (CRS040, green emitting, and Red305, red emitting), also illustrating the Stokes' shift.

emitted light in the solar cell(s) at the side(s) of the plastic body. The energy of the emitted photons ideally is only somewhat larger than the band gap of the attached solar cells, to ensure near-unity conversion efficiency, and an increase in solar cell efficiency would result. Also, it was suggested to use a number of different organic dye molecules of which the re-emitted light was matched for optimal conversion by different solar cells. This is similar to using a stack of multiple solar cells, each sensitive to a different part of the solar spectrum. Efficiencies of $\sim 30 \%$ were expected [5].

The development of LSCs began as an alternative approach to lower the costs of PV. Unlike standard solar concentrators both direct and diffuse light is concentrated, without the need for expensive tracking, by a factor of typically 5-10, and smaller silicon (or other more expensive) solar cells can be used. As the cost of the transparent plastic is expected to be much lower than the area cost of the solar cell the cost per Watt-peak (Wp) of an LSC is lower compared to the cost of a planar silicon solar cell. Also, LSCs are of special interest for building or infrastructure integrated PV applications, as illustrated in Fig. 2.
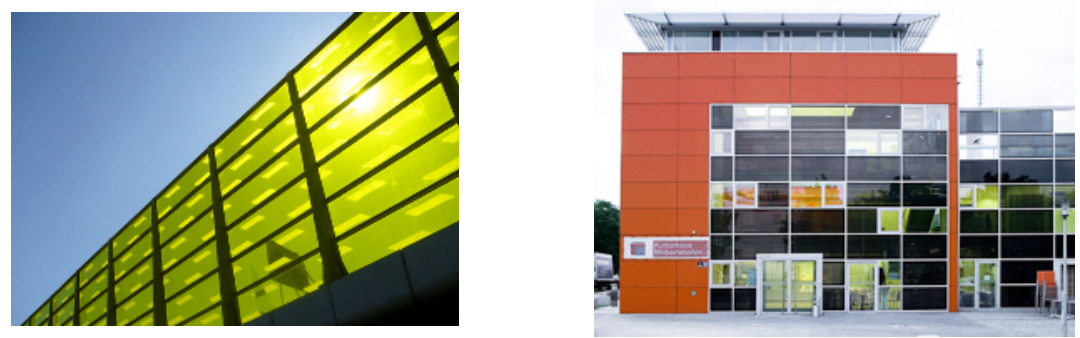

Fig. 2. (left) Example of potential application as soundbarrier; (right) example of potential application in the building envelope.

Key issues in LSC research are optimizing the absorption of luminescent species while having a small spectral overlap between absorption and emission and high luminescent quantum yield. In addition, low matrix absorption and high stability are required. Much progress has occurred, which is illustrated by the efficiency record of 7.1\% [7] for organic dyes in PMMA, as a result of modelling 


\section{$2^{\text {nd }}$ European Energy Conference}

optimization study [2] and material development. In addition, nanocrystals or quantum dots (QDs) are increasingly used replacing the organic dyes; LSCs are then referred to as Quantum Dot Concentrators (QDCs) [8].

\section{Basics}

The principle of operation of the LSC is illustrated in Fig. 1. Light is incident on the top of a plastic plate with refractive index $n$. In this plate luminescent species are present, that absorb the light and isotropically emit it at a wavelength, which is generally red shifted as a result of the Stokes' shift of the particular luminescent specie (see also Fig. 1). The Stokes' shift is defined as the difference between maxima in absorption and emission spectra, related to the same transition. Part of the emission falls within the escape cone (determined by the critical angle $\left(\theta_{c}\right)$ ) and is lost from the luminescent concentrator at the surfaces (1). The critical angle is defined by

$$
\theta_{c}=\sin ^{-1}(1 / n)
$$

The other part (2) of the luminescence is guided to the solar cell by total internal reflection, which is lossless within the angular range. The other sides of the plastic plate should be covered with mirrors to trap photons outside the angular range. The efficiency of the complete LSC including solar cell $\eta_{L S C}$ can be expressed as the product of the optical efficiency $\eta_{\text {opt }}$ of the LSC plate and the efficiency of the solar cell $\eta_{P V} ; \eta_{\text {opt }}$ is written as [9]:

$$
\eta_{\text {opt }}=(1-R) \eta_{\text {abs }} \eta_{L Q E} \eta_{S} \eta_{\text {trap }} \eta_{\text {mat }}\left(1-\eta_{\text {self }}\right) \eta_{T I R}
$$

with $R$ the surface reflection coefficient, $\eta_{a b s}$ the absorption efficiency (the fraction of sunlight absorbed by the luminescent species), $\eta_{L Q E}$ the quantum efficiency of the luminescent species, $\eta_{S}$ the Stokes' efficiency, which is the energy loss between absorption and emission, $\eta_{\text {trap }}$ the efficiency of trapping of light (fraction of light outside the escape cone), $\eta_{\text {mat }}$ the efficiency of transmission of light in the plastic matrix, $\eta_{\text {self }}$ the efficiency of self absorption of the luminescent species, $\eta_{T I R}$ the efficiency of total internal reflection.

The Fresnel reflection loss equals $[(n-1) /(n+1)]^{2}$. For a polymethylmethacrylate (PMMA) plastic with $n=1.49$ this loss amounts to 0.0387 ; this can be lowered by applying an anti-reflection coating. The fraction of sunlight that can be absorbed by the luminescent species depends on the specific material; organic dyes have narrow absorption bands, in contrast to QDs. An example is given in Fig. 1, in which the absorption and emission spectra are depicted for Fluorescence Yellow CRS040 (coumarine dye, from Radiant Color) and Lumogen F Red305 (perylene, from BASF). The loss due to non-unity quantum efficiency is typically low for organic dyes, while QDs may have quantum efficiency values between $20-80 \%$. The Stokes' shift between absorption and emission is required to be small to minimize energy loss; on the other hand it should be large to minimize the overlap between the emission and absorption spectrum causing re-absorption (or self-absorption). The light trapping efficiency can be written as $(1 / n) \sqrt{ }\left(n^{2}-1\right)$, which equals 0.741 for PMMA; the critical angle is $\theta_{c}=42.2^{\circ}$. This efficiency can be enhanced by the use of selective mirrors or mirrors based on photonic materials. The transmission efficiency in the matrix depends on scattering or absorption in the matrix; an absorption coefficient of $1 \mathrm{~m}^{-1}$ is typical. Self-absorption in general leads to a red-shift of the emission spectrum, as well as intensity loss. Total internal reflection loss depends on surface quality. Taking into account all these losses, typical LSC efficiencies range from 3-8 \%.

The maximum attainable LSC efficiency was determined using the Shockley-Queisser principle of detailed balance. Efficiency values as high as 33\% were calculated, due to the presence of a perfect band pass mirror at the top surface [10]. The maximum concentration $(C)$ possible has been determined to be dependent on Stokes' shift of the luminescent species. For the Lumogen F Red 300 dye from BASF it can be calculated that $C=119$, as absorption and emission maxima are 578 and 613 $\mathrm{nm}$, respectively [5]. This maximum is one order of magnitude larger than typically used in practical LSCs. 


\section{Design modeling}

Two different approaches are used for the modelin of LSCs, a thermodynamic detailed balance model which is based on the radiative energy transfer between mesh points in the concentrator plate [8], and a ray-tracing model in which every incoming photon is tracked and its fate determined [11]. Both methods yield similar results, and are also in excellent agreement with experiments on test devices. With the thermodynamic model, requiring only a small amount of input parameters, results can be obtained quickly; however geometries are limited to block shapes only. In addition, only single absorbing species can be modelled presently. Ray-trace models are computationally expensive, as a large number of luminescent species have to be considered, as well as a large number of rays to obtain accurate results; however, simpler procedures involving absorption coefficients allow for much faster modelling.

Ray-tracing has been used to perform a parametric analysis for a $5 \times 5 \mathrm{~cm}^{2}$ planar LSC (with a c-Si solar cell on one side, and mirrors at the remaining side and bottom surfaces) to find attainable LSC efficiencies for various mirror configurations, plastic matrix material properties, type of solar cell and dye. The concentrator consists of a PMMA plate (refractive index $n=1.49$, absorption $1.5 \mathrm{~m}^{-1}$ ) doped with two luminescent dyes, CRS040 from Radiant Color and Lumogen F Red 305 from BASF (Fig. 1), with a luminescent quantum efficiency of $95 \%$. The conversion efficiency of this LSC was modelled to be $2.45 \%$ [2].

Direct mirrors or a mirror with an air-gap between the mirror and the LSC has been modelled, as well as different reflectivity values and specular or Lambertian mirror types. Without a side mirror, rays within the escape cone leave the LSC at that side; rays outside the escape cone are subject to total internal reflection. When a direct mirror is applied, the total internal reflection disappears, and all rays reflect with the reflection coefficient of the mirror. For the rays outside the escape cone this leads to a reduction in the reflection, and thus lower power conversion efficiency. The use of an "airgap mirror", i.e., an air-gap between mirror and LSC, combines total internal reflection with reflection of the escaping rays. The results of the calculations (Table 1) show a maximum efficiency of $2.94 \%$ for the case with $97 \%$ reflective air-gap mirrors (e.g. $3 \mathrm{M}$ adhesive visible mirror foil, with a reflection of 97\%) at the sides and a 97\% reflective Lambertian air-gap mirror (e.g. standard integrating sphere material with a reflection of $97 \%$ ) at the bottom.

In a similar manner the background absorption of the polymer was varied in the absorption and emission range of the dye from the current value of $1.5 \mathrm{~m}^{-1}$ to a value of $10^{-3} \mathrm{~m}^{-1}$. It was found that the efficiency of the LSC increased to slightly over $3.4 \%$. An additional increase of the refractive index of the polymer from 1.49 to 1.7 would result in an increase in efficiency to almost $3.8 \%$, as a result of the fact that a higher refractive index leads to a larger fraction of the emitted dye luminescence being trapped in the concentrator plate. Note, however, that a polymer with the combination of a refractive index of 1.7 and an optical absorption of $10^{-3} \mathrm{~m}^{-1}$ is not currently available.

Table 1. Calculated efficiencies (in \%) for an LSC for various optimized configurations and parameters [2].

\begin{tabular}{|c|c|c|c|}
\hline c-Si & GaAs & InGaP & parameters \\
\hline 2.4 & 4.2 & 5.9 & fixed mirrors, 85\% reflectivity, dyes with 95\% LQE \\
\hline 2.9 & 5.1 & 7.1 & $\begin{array}{c}97 \% \text { reflectivity “air-gap mirrors" on sides, and } \\
97 \% \text { reflectivity Lambertian mirror at bottom }\end{array}$ \\
\hline 3.4 & 5.9 & 8.3 & $\begin{array}{c}\text { reduce background absorption of polymer matrix } \\
\text { from } 1.5 \mathrm{~m}^{-1} \text { to } 10^{-3} \mathrm{~m}^{-1}\end{array}$ \\
\hline 3.8 & 6.5 & 9.1 & \begin{tabular}{c} 
increase of refractive index from 1.49 to 1.7 \\
\hline
\end{tabular} \\
\hline
\end{tabular}




\section{$2^{\text {nd }}$ European Energy Conference}

One major reason for the lower efficiency compared to early work is that the used solar cells are not optimized for the emission-spectrum of the dye: c-Si solar cells absorb all the light up to about $1100 \mathrm{~nm}$, but the dyes used in these LSCs emit around $650 \mathrm{~nm}$. It would be beneficial to use a solar cell with a larger band gap, because these deliver the same current as the mc-Si cell, but at larger open circuit voltage $\left(V_{o c}\right)$. In Table 1 estimates are listed for attainable efficiencies for similar concentrator plates with different solar cells attached. Replacing the c-Si cell with a GaAs or InGaP cell, will increase $\eta$ from $3.8 \%$ to 6.5 and $9.1 \%$, respectively (based on $V_{o c}$ (fillfactor) values of 0.58 $\mathrm{V}(0.83), 1.00 \mathrm{~V}(0.83)$, and $1.38 \mathrm{~V}(0.84)$, for c-Si, GaAs, InGaP, respectively). Thus, the use of GaAs or InGaP cells will result in higher efficiencies, albeit at higher cost. A cost calculation must be performed to determine if the combination of the luminescent concentrator with this type of cells is an interesting alternative to c-Si based solar technology. Note that using the two-dye combination and the InGaP cell still leaves a large part of the solar spectrum unabsorbed. Extending the absorption range of the luminescent species to $\sim 1000 \mathrm{~nm}$ would bring LSC efficiency above $10 \%$.

The effect of varying the device geometry on LSC performance has been modeled by Kennedy et al. [12]. Square, right-angled triangular and hexagonal quantum dot doped luminescent solar concentrators of increasing top surface apertures $\left(A_{\text {conc }}\right)$ were considered. Concentration ratios $(C)$ were predicted for increasing $A_{\text {conc }}$ of each geometry type. It was found that a hexagonal geometry attains the highest $C$ for the range of $A_{\text {conc }}$ considered. However, for a given $A_{\text {conc }}$, each geometry type has a different area of the attached solar cell $\left(A_{p v}\right)$. Also, the cost of materials of the LSC plate, although much lower than the cost of the solar cell $\left(\right.$ per $\mathrm{m}^{2}$ ), cannot be neglected. Therefore, to determine the optimum geometry, relative costs per unit power output have been calculated, assuming that the cost ratio of plate and solar cell is $1 / 20$. The relative power output of each device is assumed to be proportional to the product of the concentration ratio attained and $A_{p v}$. The results indicate that all geometries can attain the same minimum relative cost per unit power, however with differing $A_{\text {conc }}$.

Others have also modeled LSC design: Currie et al. [13] projected conversion efficiencies as high as $6.8 \%$, for a tandem LSC based on two single LSCs that consist of thin layer of deposited organic dye molecules onto a glass plate to which a GaAs cell was attached. Using $\mathrm{CdTe}$ or $\mathrm{Cu}(\mathrm{In}, \mathrm{Ga}) \mathrm{Se}_{2}$ solar cells conversion efficiencies of $11.9 \%$ and $14.5 \%$, respectively, were calculated. In contrast, Farrell and Yoshida [14] recently argued that so-called first generation LSCs can only attain efficiencies larger than $10 \%$ for very small concentration ratios. Only second generation LSCs, in which escape cone losses and reabsorption losses are minimized, could attain efficiencies that approach the $30 \%$ value as reported by Smestad et al. [5].

Annual performance has been modeled using an LSC of which the properties and geometry resulted from a cost-per-unit-of-power optimization study [15]. A square plate of $23.7 \times 23.7 \times 0.1 \mathrm{~cm}^{3}$ was used and in the ray-trace model attached to four c-Si solar cells (18.59\% efficiency) on all sides. Using a Lumogen F Red 305 (BASF) dye it was calculated that $46.5 \%$ of all photons in the wavelength range of $370-630 \mathrm{~nm}$ were collected, leading to an LSC efficiency of $4.24 \%$ [2]. The annual yield of this LSC was determined using realistic spectra representative for the Netherlands [16] and amounted to $41.3 \mathrm{kWh} / \mathrm{m}^{2}$, which is equivalent to an effective annual efficiency of $3.81 \%$.

\section{State-of-the-art devices}

Recent efforts to surpass the historical 4\% efficiency limit of LSCs [9] albeit for smaller area size, have been successful. For example, Goldschmidt et al. [17] showed for a stack of two plates with different dyes, to which four GaInP solar cells were placed at the sides, that the conversion efficiency is $6.7 \%$; the plate was small $\left(4 \mathrm{~cm}^{2}\right)$, and the concentration ratio was only 0.8 . It was argued that the conversion efficiency was limited by the spectral range of the organic dyes used, and that if the same quantum efficiency as was reached for the 450-600 $\mathrm{nm}$ range could be realized for the range $650-1050 \mathrm{~nm}$ an efficiency of $13.5 \%$ could be within reach. They also discuss the benefits of a photonic structure on top of the plate, to reduce the escape cone loss [17]. The proposed structure is a so-called rugate filter; this is characterized by a varying refractive index in contrast to 
standard Bragg reflectors, which suppresses the side loops that could lead to unwanted reflections. The use of these filters would increase the efficiency by $\sim 20 \%$, as was determined for an LSC consisting of one plate and dye. Slooff et al. [7] presented results on 50x50x5 mm $\mathrm{m}^{3}$ PMMA plates in which both CRS040 and Red305 dyes were dispersed at 0.003 and $0.01 \mathrm{wt} \%$, respectively. The plates were attached to either mc-Si, GaAs or InGaP cells, and a diffuse reflector (97\% refection) was used at the rear side of the plate. The highest efficiency measured was $7.1 \%$ for $4 \mathrm{GaAs}$ cells connected in parallel (7\% if connected in series). The external quantum efficiency of the LSC between $400-600 \mathrm{~nm}$ results from dye absorption in that wavelength range and equals $23-25 \%$ for the c-Si and GaAs and $\sim 9 \%$ for the InGaP cell. Similar plates were attached to a-Si:H solar cells, but the attained efficiency was $\sim 1 \%$ for two cells connected in parallel [18].

Quantum dots are potential candidates to replace organic dye molecules in an LSC, for their higher brightness, better stability, and wider absorption spectrum [19]. Schüler et al. [20] proposed to make LSCs by coating transparent glass substrates with QD-containing composite films, using a potentially cheap sol-gel method. They reported on the successful fabrication of thin silicon oxide films that contain CdS QDs using a sol-gel dip-coating process, whereby the 1-2 $\mathrm{nm}$ sized CdS QDs are formed during thermal treatment after dip-coating. Depending on the anneal temperature, the colors of the LSC ranged from green for $250{ }^{\circ} \mathrm{C}$ to yellow for $350{ }^{\circ} \mathrm{C}$ and orange for $450{ }^{\circ} \mathrm{C}$. Reda [21] also prepared CdS QD concentrators, using sol-gel spin coating, followed by annealing. The annealing temperature was found to affect absorption and emission spectrum: luminescent intensity and Stokes' shift both decreased for 4 weeks outdoor exposure to sunlight, which probably was caused by aggregation and oxidation. It is known that oxidation leads to blue-shifts in emission [22]. Blue-shifts have also been observed by Gallagher et al. [23] who dispersed CdSe QDs in several types of resins (urethane, PMMA, epoxy), for fabrication of LSC plates.

Bomm et al. [24] have addressed several problems regarding incorporation of QDs in an organic polymer matrix, viz. phase separation, agglomeration of particles leading to turbid plates, and luminescence quenching due to exciton energy transfer. They have synthesized QDSCs using CdSe core/multishell QDs $(\mathrm{QE}=60 \%)$ [25] that were dispersed in laurylmethacrylate (LMA). UVpolymerization was employed to yield transparent PLMA plates with QDs without any sign of agglomeration. To one side of this plate, a mc-Si solar cell was placed, and aluminum mirrors to all other sides. The best QDC made generated a current of $77.14 \mathrm{~mA} / \mathrm{cm}^{2}$, nearly twice as much as the bare cell $\left(5 \times 0.5 \mathrm{~cm}^{2}\right)$ that generated a current density of $40.28 \mathrm{~mA} / \mathrm{cm}^{2}$ at the $1000 \mathrm{~W} \mathrm{AM} 1.5 \mathrm{G}$ spectrum. The QDC efficiency is 3.5\%. Besides QDs also nanorods (NRs) have been dispersed in PLMA, showing excellent transmittance of 93\%; for long rods (aspect ratio of 6) a QE of $70 \%$ is observed, which is only slightly smaller than the QE in solution, implying that these rods are stable throughout the polymerization process.

Hyldahl et al. [26] used commercially available $\mathrm{CdSe} / \mathrm{ZnS}$ core/shell QDs with QE=57\% in LSCs, both liquid (QDs dissolved in toluene, between two $6.2 \times 6.2 \times 0.3 \mathrm{~cm}$ glass plates) and solid (QDs dispersed in epoxy), and they obtained an efficiency of $3.98 \%$ and $1.97 \%$, respectively. They also used the organic dye Lumogen F Red300, and obtained efficiency of $2.6 \%$ in toluene.

\section{Cost}

Widespread deployment of LSCs (or any next generation PV technology) will only be possible if the cost per Watt-peak generated is lower than $1 € / \mathrm{Wp}$. LSC cost is composed of plate cost and solar cell cost, which are related to their respective areas, and thus are detailed in $€ / \mathrm{m}^{2}$. Currie et al. [13] use cost in $€ / \mathrm{Wp}$, and they find that using a $50 \$ / \mathrm{Wp}$ GaInP solar cell the geometrical gain should be as high as 50. Hence, both solar cell cost should come down as well as geometrical gain increased; following Farrell, this may only be possible with second generation LSCs. Bende et al. [15] have presented a cost-optimization study focusing on relative area cost and relative cost per unit of power, since they compare LSC with conventional PV cost. Assuming a realistic ratio between polymerplate cost and PV area cost of $1 / 15$, they found that the lowest relative cost per unit of power can be as low as 0.35 to 0.55 for a combined plate thickness and length of $1 \mathrm{~mm}$ and $0.25 \mathrm{~m}$, and $10 \mathrm{~mm}$ 


\section{$2^{\text {nd }}$ European Energy Conference}

and $0.55 \mathrm{~m}$, respectively. Thus, LSC cost could be $\sim 1 / 3$ of c-Si cost, in terms of relative cost per unit of power. As these optimized costs depend critically on the ratio between polymer-plate cost and PV area cost, a sensitivity study showed that this ratio should at least be lower than $1 / 5$ for LSCs to be economically viable. Note, Kennedy et al. [12] used a ratio of 1/20.

\section{Outlook}

LSC development will need to focus on material systems that: 1) absorb all photons with wavelength $<950 \mathrm{~nm}$, and emit them red-shifted at $\sim 1000 \mathrm{~nm}$, for use with c-Si solar cells; alternatively, stacked plates with matched cells [3] may be developed that effectively also absorb these photons; 2) have as low as possible spectral overlap between absorption and emission spectra to minimize re-absorption losses; 3) have near unity luminescence quantum yield; 4) have low escape cone losses; 5) be stable outdoors for more than 10 years; 6) be easy to manufacture at low cost [27]. The present lack of NIR dyes will prohibit further increase of the present $7.1 \%$ record conversion efficiency towards the $30 \%$ limit. Here, quantum dots or nanorods may have to be used, as their broad absorption spectrum is very favourable. However, they should be emitting in the NIR at high quantum efficiency, larger than the present $\sim 70-80 \%$, and their Stokes' shift should be larger. Another strategy could be the use of so-called type II QDs, as their Stokes' shift could be very large $(\sim 300 \mathrm{~nm})$, however, but their stability and QE are not good enough yet. Modeling results indicate that using type II QDs could lead to an efficiency of $7.5 \%$ for a $50 \times 50 \times 5 \mathrm{~mm}^{3}$ QDSC compared to a $5 \%$ efficient LSC with Red305 dye [28]. Stability could be improved using multishell QDs [25] while interfacial alloying can be optimized to obtain type II QDs with desired properties, i.e., a Stokes' shift of $50-100 \mathrm{~nm}$, without spectral overlap. An different approach was presented recently that employs resonanceshifting to circumvent reabsorption losses [29]. Alternatively, the originally proposed three-plate stack [3] could be further developed using perhaps a combination of organic dyes and nanocrystals, or even rare earth ions [27], with optimized dedicated solar cells for each spectral region.

\section{Conclusion}

In this contribution an short overview of R\&D on LSCs was presented. The renewed interest in the LSCs, since the pioneering work in the 1970s and 1980s, has lead to the development of new materials and device concepts: new dyes, and more importantly, new quantum dots have been developed and have been included in the polymer concentrators. Stability tests for over 2 years have shown that good candidates exist for use in commercial type LSCs. Future research should be directed towards lowering escape cone losses, lowering the absorption in the matrix, as well as reabsorption, i.e., second generation LSCs. Further, the absorption range should be enlarged in order to realize efficiencies well above $10 \%$; this may be possible using appropriately tuned quantum dots in a single plate configuration, or multi plate hybrid stacks in which both organic and inorganic materials are combined.

\section{Acknowledgements}

The author gratefully acknowledges numerous colleagues at Utrecht University and elsewhere who contributed to the presented work. Part of this work was financially supported by the European Commission as part of the Framework 6 integrated project FULLSPECTRUM (contract SES6-CT2003-502620), SenterNovem as part of their Netherlands Nieuw Energie Onderzoek (New Energy Research) programme, Netherlands Foundation for Fundamental Research on Matter (FOM), and Netherlands Organisation for Scientific Research (NWO). 


\section{References}

1. EPIA (European Photovoltaic Industry Association), Market report 2011 (EPIA, Brussel, 2012).

2. W.G.J.H.M. Van Sark, K.W.J. Barnham, L.H. Slooff, A.J. Chatten, A. Büchtemann, A. Meyer, S.J. McCormack, R. Koole, D.J. Farrell, R. Bose, E.E. Bende, A.R. Burgers, T. Budel, J. Quilitz, M. Kennedy, T. Meyer, A. Meijerink, D. Vanmaeckelbergh, Optics Express, 16 (2008) 21773 21792

3. A. Goetzberger, W. Greubel, Applied Physics, 14 (1977) 123-139

4. W.H. Weber, J. Lambe, Applied Optics, 15 (1976) 2299-2300

5. G. Smestad, H. Ries, R. Winston, E. Yablonovitch, Solar Energy Materials, 21 (1990) 99-111

6. B.C. Rowan, L.R. Wilson, B.S. Richards, IEEE Journal of Selected Topics in Quantum Electronics, 14 (2008) 1312-1322

7. L.H. Slooff, E.E. Bende, A.R. Burgers, T. Budel, M. Pravettoni, R.P. Kenny, E.D. Dunlop, A. Büchtemann, physica status solidi (RRL), 2 (2008) 257-259

8. A.J. Chatten, K.W.J. Barnham, B.F. Buxton, N.J. Ekins-Daukes, M.A. Malik, Semiconductors, 38 (2004) 609-617

9. V. Petrova-Koch, A. Goetzberger (eds.) High-Efficient Low-Cost Photovoltaics - Recent Developments (Springer, Heidelberg, 2008)

10. U. Rau, F. Einsele, G.C. Glaeser, Applied Physics Letters, 87 (2003) 171101

11. A.R. Burgers, L.H. Slooff, R. Kinderman, J.A.M. van Roosmalen, Proceedings of the 20th European Photovoltaic Solar Energy Conference (2005) 394-397

12. M. Kennedy, S. J. McCormack, J. Doran, B. Norton, Proceedings of ISES World Solar Congress (2007).

13. M.J. Currie, J.K. Mapel, T.D. Heidel, S. Goffri, M.A. Baldo, Science, 321 (2008) 226-228.

14. D.J. Farrell, M. Yoshida, Progress in Photovoltaics: Research and Applications, 20 (2011) 9399.

15. E.E. Bende, A.R. Burgers, L.H. Slooff, W.G.J.H.M. Van Sark, M. Kennedy, Proceedings of Twenty third European Photovoltaic Solar Energy Conference (2008) 461-469

16. B. Houshyani Hassanzadeh, A.C. De Keizer, N.H. Reich, W.G.J.H.M. Van Sark, Proceedings of 22nd European Photovoltaic Solar Energy Conference (2007) 2652-2658

17. J.C. Goldschmidt, M. Peters, A. Bösch, H. Helmers, F. Dimroth, S.W. Glunz, G. Willeke, Solar Energy Materials and Solar Cells, 93 (2009) 176-182.

18. D.J. Farrell, W.G.J.H.M. van Sark, S.T. Velthuijsen, R.E.I. Schropp, physica status solidi C, 7 (2009) 1045-1048.

19. K. Barnham, J.L. Marques, J. Hassard, P. O'Brien, Applied Physics Letters, 76 (2000) $1197-$ 1199

20. A. Schüler, M. Python, M. Valle del Olmo, E. de Chambrier, Solar Energy, 81 (2007) 11591165

21. S.M. Reda, Acta Materialia, 56 (2008) 259-264

22. W.G.J.H.M. Van Sark, P.L.T.M. Frederix, A.A. Bol, H.C. Gerritsen, A. Meijerink, ChemPhysChem, 3 (2002) 871-879

23. S.J. Gallagher, B.C. Rowan, J. Doran, B. Norton. 2007. Solar Energy, 81 (2007) 540

24. J. Bomm, A. Büchtemann, A.J. Chatten, R. Bose, D.J. Farrell, N.L.A. Chan, Y. Xiao, L.H. Slooff, T. Meyer, A. Meyer, W.G.J.H.M. van Sark, R. Koole, Solar Energy Materials and Solar Cells, 95 (2011) 2087-2094

25. R. Koole, M. Van Schooneveld, J. Hilhorst, C. de Mello Donegá, D.C. 't Hart, A. van Blaaderen, D. Vanmaekelbergh, A. Meijerink, Chemistry of Materials, 20 (2008) 2503-2512

26. M.G. Hyldahl, S. T. Bailey, B. P. Wittmershaus. Solar Energy, 83 (2009) 566-573

27. B.C. Rowan, L.R. Wilson, B.S. Richards. IEEE Journal of Selected Topics in Quantum Electronics, 14 (2008) 1312-1322

28. W.G.J.H.M. van Sark, C. de Mello Donegá, R.E.I. Schropp, Advances in Science and Technology, 74 (2010) 176-181

29. N.C. Giebink, G.P. Wiederrecht, M.R. Wasielewski, Nature Photonics 5, (2011) 694-701 